LUNG TRANSPLANTATION

\title{
Prevalence and clonality of Burkholderia cepacia complex genomovars in UK patients with cystic fibrosis referred for lung transplantation
}

\author{
A De Soyza, K Morris, A McDowell, C Doherty, L Archer, J Perry, J R W Govan, P A Corris, \\ K Gould
}

Thorax 2004;59:526-528. doi: 10.1136/thx.2003.010801

See end of article for
authors' affiliations
....................
Correspondence to:
Dr A De Soyza, William
Leech Centre, The Freeman
Hospital, High Heaton,
Newcastle upon Tyne NE7
7DN, UK; anthony
de-soyza@ncl.ac.uk
Received 16 June 2003
Accepted
12 December 2003

\begin{abstract}
Background: It has previously been reported that patients infected with Burkholderia cenocepacia (genomovar III) before lung transplantation have a poorer outcome than those with other B cepacia complex infections.

Methods: An extensive study was conducted to determine the prevalence and clonality of $B$ cepacia complex genomovars isolated from patients referred for transplant assessment between 1989 to the present and, where appropriate, whether strain type was related to transplant outcome.

Results: Isolates from 29 patients were identified as $B$ cepacia complex organisms by molecular analysis. Thirteen patients $(45 \%)$ were infected with the highly transmissible ET-12 strain of $B$ cenocepacia recA lineage III-A, while all remaining patients were infected with genetically unique $B$ cenocepacia, $B$ multivorans, and $B$ vietnamiensis strains. All previously reported deaths following transplantation were associated with ET-12 infection.

Conclusions: The ET-12 strain is the predominant cause of $B$ cenocepacia infections in patients with cystic fibrosis referred to our pulmonary transplant centre and is associated with poor transplant outcomes using standard treatment regimens.
\end{abstract}

B urkholderia cepacia is a life threatening pathogen in patients with cystic fibrosis (CF). The unique clinical syndrome of necrotising pneumonia and associated septicaemia known as "cepacia syndrome" is a feared complication of infection. ${ }^{1} B$ cepacia infections have also proved highly transmissible leading to epidemics within many CF centres. ${ }^{2}$

Polyphasic taxonomic studies have shown that isolates previously classified as $B$ cepacia comprise at least nine phenotypically similar genomovar species. ${ }^{2}$ This group of organisms is collectively known as the Burkholderia cepacia complex. Analysis of the recA housekeeping gene has revealed that strains of $B$ cenocepacia (formerly genomovar III) can be split into four distinct groups known as III-A, III-B, III-C, and III-D. ${ }^{3}$ The clinical significance of these $B$ cenocepacia subgroups is, however, unclear. Epidemiological studies have found a disproportionate distribution of $B$ cepacia complex genomovars among patients, with $B$ cenocepacia and, to a lesser extent, $B$ multivorans (formerly genomovar II) the most prevalent organisms. ${ }^{2}$ Most cases of "cepacia syndrome" are associated with $B$ cenocepacia, although it has been unclear if this represents increased virulence or simply its high prevalence among patients. However, retrospective studies have shown that CF patients with $B$ cenocepacia infections before transplantation have poorer post-transplant outcomes than patients infected with other genomovars, providing evidence that $B$ cenocepacia is, on the whole, a more aggressive and virulent organism. ${ }^{4} 5$

Data on $B$ cenocepacia specific virulence and transmissibility factors is at best limited. The $B$ cenocepacia epidemic strain known as ET-12 is characterised by the expression of the $c b l A$ gene. ${ }^{6}$ In addition, a conserved $1.4 \mathrm{kbp}$ DNA fragment, the $B$ cepacia epidemic strain marker (BCESM), has been described. ${ }^{7}$ The ET-12 lineage is unique in bearing both the cblA gene and BCESM. ${ }^{2}$ However, outbreaks have been observed involving strains that lack the cblA gene or both putative transmissibility markers. ${ }^{8}$

Our transplant unit serves CF units in Northern England, Scotland and both Northern and Southern Ireland. We conducted an extensive epidemiological study to determine the prevalence and clonality of isolates in these regions since 1989. B cenocepacia strain types were investigated for the presence of epidemic clones as well as putative transmissibility markers, and these findings were related to transplant outcome.

\section{METHODS \\ Case finding}

A retrospective review of the pulmonary transplant database at the Freeman Hospital from 1989 (programme start) to 2002 was performed. The management of transplant patients was as previously published. ${ }^{9}$

\section{Microbiology}

Sputum was collected from patients during pre-transplant assessment and immediately before surgery. Seven days after transplantation bronchoalveolar lavage fluid was collected from recipients and presumed $B$ cepacia complex bacteria were isolated by culture. Phenotypic analyses were performed using the API 20NE diagnostic test (Biomèrieux, Marcy l'Etoile, France).

\section{PCR based analyses}

Genomic template DNA for molecular analysis was prepared from all isolates and their genomovar status determined using rec $A$ based methods. ${ }^{10}$ Analysis of PCR and RFLP products by agarose electrophoresis was as previously described. ${ }^{10}$ PCR based detection of the BCESM and the cbla gene was conducted as previously reported. ${ }^{71}$ 
Pulsed field gel electrophoresis

$B$ cepacia complex strains were genotyped by macrorestriction of whole genomic DNA with the restriction enzyme SpeI (New England Biolabs, UK) and the fragments were separated by pulse field gel electrophoresis (PFGE) (CHEF DRII System; Bio-Rad). Genotype patterns for $B$ cenocepacia isolates were compared with those of the ET-12 index strain J2315. Investigators were blinded to the genomovar status of the $B$ cepacia complex strains isolated.

\section{RESULTS}

Prevalence of $B$ cepacia complex genomovars in CF patients

Thirty two patients referred to the Freeman Hospital Transplant Unit were found to have presumptive $B$ cepacia complex infection based on phenotypic analysis. However, further analysis revealed that isolates from one patient were Brevundimonas vesicularis, while isolates from two patients were identified as Alcaligenes xylosoxidans. Sixteen of the remaining 29 patients ( $55 \%$ ) were found to have $B$ cenocepacia infections (14 (48\%) B cenocepacia III-A and two $(7 \%) B$ cenocepacia III-B), 11 patients $(38 \%)$ were infected with $B$ multivorans, while the remaining two patients $(7 \%)$ had $B$ vietnamiensis (formerly genomovar $\mathrm{V}$ ) infections.

\section{Molecular epidemiology of B cenocepacia infection}

Four strain types were found among the 16 patients infected with $B$ cenocepacia (fig 1). Thirteen patients were infected with the $B$ cenocepacia ET-12 epidemic strain. All ET-12 isolates contained both the cblA gene and BCESM. The three remaining $B$ cenocepacia III-A and III-B strains were genetically unique. The unique $B$ cenocepacia III-A strain lacked the cblA gene but was positive for BCESM. PFGE analysis of ET12 isolates before and after transplantation from two representative patients revealed the persistence of the epidemic strain after surgery (fig 2).

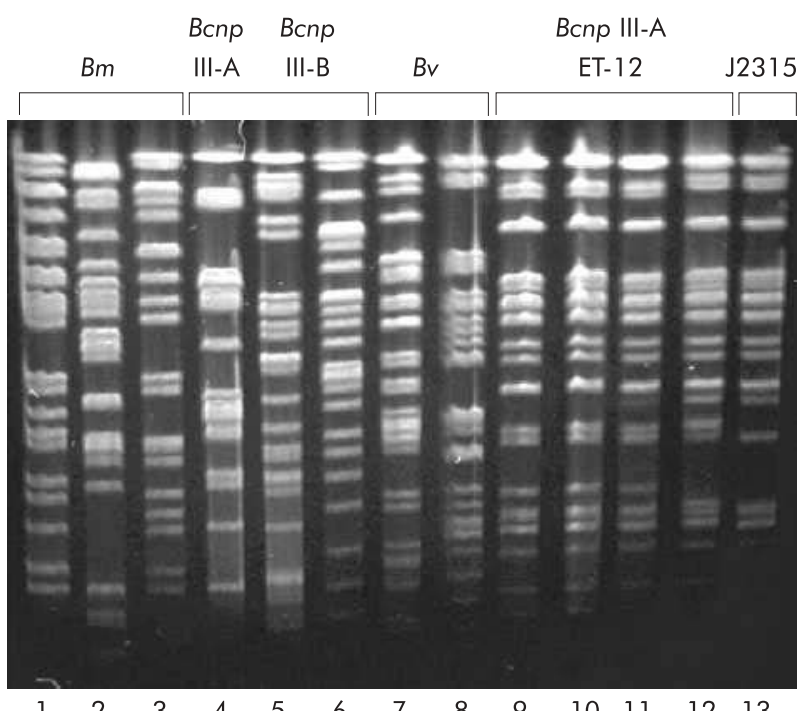

Figure 1 Macrorestriction (Spel) fingerprinting of $B$ cepacia complex genomovar strains recovered from patients with CF attending The Freeman Hospital Transplant Unit. Lanes 1-3, representative sample of unique $B$ multivorans $(B m)$ strains; lane 4 , unique $B$ cenocepacia III-A (Bcnp III-A) strain; lanes 5-6, unique $B$ cenocepacia III-B (Bcnp III-B) strains; lanes 7-8, unique $B$ vietnamiensis $(B v)$ strains; lanes 9-12, representative sample of $B$ cenocepacia III-A ( $B \subset n p$ III-A) ET-12 isolates; lane 13, J2315 Edinburgh index strain for ET-12 lineage. Restriction fragments were separated using pulse times of 2.9-35.4 seconds at $6 \mathrm{~V} / \mathrm{cm}$ for 20 hours at $14^{\circ} \mathrm{C}$.

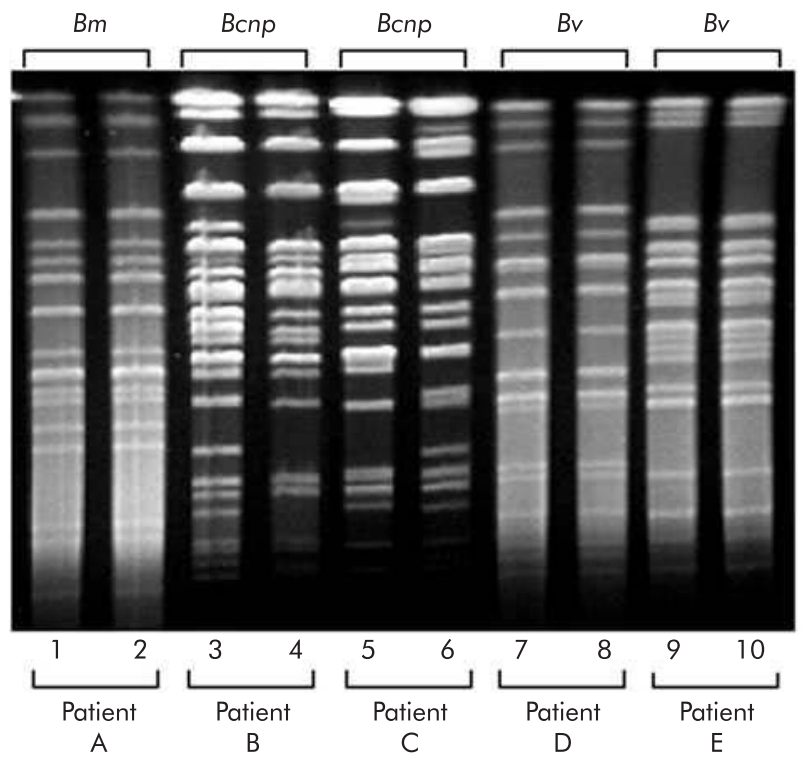

Figure 2 Persistence of pre-transplant $B$ cepacia complex strains in post-transplant cultures by macrorestriction (Spel) fingerprinting. Preand post-transplant isolates from patient $A$ (lanes 1-2: $B$ multivorans, $B m$ ), patient $B$ (lanes 3-4: $B$ cenocepacia III-A ET-12, Bcnp), patient $C$ (lanes 5-6: B cenocepacia III-A ET-12; Bcnp), patient D (lanes 7-8: B vietnamiensis, $B v$ ), and patient $E$ (lanes 9-10: $B$ vietnamiensis, $B v$ ). Restriction fragments were separated using pulse times of 2.935.4 seconds at $6 \mathrm{~V} / \mathrm{cm}$ for 20 hours at $14^{\circ} \mathrm{C}$.

\section{Molecular epidemiology of $B$ multivorans and $B$ vietnamiensis infections}

All $B$ multivorans and $B$ vietnamiensis strains were found to be unique by PFGE (fig 1). All strains were negative for the $c b l A$ gene and BCESM. PFGE analysis of pre- and post-transplant $B$ multivorans and $B$ vietnamiensis isolates again revealed the persistence of the infecting strain after surgery (fig 2).

\section{Strain types and post-transplant outcomes}

PFGE analysis also revealed that all our previously described post-transplant deaths ${ }^{4}$ were due to infections with the $B$ cenocepacia ET-12 epidemic strain. The previously described patient group who were successfully transplanted all had pretransplant infections due to genetically unique strains of $B$ multivorans $(\mathrm{n}=3)$ and $B$ vietnamiensis $(\mathrm{n}=2)$. These patients are all currently alive and well. Four of our post-transplant survivors had $B$ multivorans or $B$ vietnamiensis organisms in the BAL fluid for up to 1 year after surgery.

\section{DISCUSSION}

Pulmonary transplantation has emerged as a highly successful treatment for end stage CF associated lung disease. There has been much debate regarding the role of transplantation in those infected with $B$ cepacia complex, reflecting the variable outcomes seen in these patients. Many transplant units consider that infection with $B$ cepacia complex before transplantation is an absolute contraindication for surgery. Currently, prevalence rates of $B$ cepacia complex infection among CF patients transplanted at this unit are $15 \%{ }^{4}$

We have previously reported that poor outcomes following transplantation were associated with pre-transplant $B$ cenocepacia infections, while infections with other genomovars were associated with excellent post-transplant prognoses, ${ }^{4}$ providing an insight into the variable outcomes previously observed in transplant patients with "B cepacia". Data supporting our observations were also reported from North Carolina, USA. ${ }^{5}$ Both studies provided evidence that the 
moratorium placed against transplanting all CF patients infected with the $B$ cepacia complex was not justified.

In this follow up study we have determined the prevalence and clonality of $B$ cepacia complex isolates recovered from patients with CF referred for pre-transplant assessment from 1989 to the present. Selective culture and phenotypic analysis identified 32 patients with $B$ cepacia complex infections, but PCR based molecular analyses revealed that only 29 were actually infected with $B$ cepacia complex pathogens. False positive rates of identification highlight the importance of molecular analyses for accurate identification of $B$ cepacia complex organisms.

Genotyping analysis in this study revealed the persistence of infection with the pre-transplant strain responsible for post-transplant infections, confirming the previous study by Steinbach et al. ${ }^{12}$ This persistent infection may reflect, in part, the difficulty in clearing the fused pleural spaces often seen during surgery. An alternative explanation for the persistence of the pre-transplant strain may be ongoing paranasal sinus infection leading to infection of the graft. In this study we found that the majority of patients referred to our unit infected with the $B$ cepacia complex were infected with $B$ cenocepacia. Analysis of these isolates by PFGE revealed that 13 patients from geographically diverse CF centres were infected with the $B$ cenocepacia III-A ET-12 clone. In contrast to the UK, the ET-12 strain is found infrequently in CF patients in the USA.

Our genotyping studies show that all our previously described post-transplant deaths related to infection with $B$ cepacia complex were associated with the ET-12 strain of $B$ cenocepacia III-A. Notably, the North Carolina study showed that poor transplant outcomes were associated with a variety of cblA negative, non-ET-12 B cenocepacia strains. ${ }^{5}$ A separate study at that US centre found that most of the 56 patients assessed for transplantation who were infected with $B$ cepacia complex harboured strains with unique genotypes. ${ }^{13}$

Since our discovery that the ET-12 strain of $B$ cenocepacia III-A was associated with poor post-transplant survival and was the most prevalent strain among $B$ cenocepacia infected patients awaiting transplantation, we have altered our previously described management of transplant patients infected with $B$ cenocepacia. ${ }^{9}$ We now omit $\mathrm{T}$ cell ablation at induction, reduce target trough cyclosporin levels, wash out the pleural cavities with the surface disinfectant taurolidine, ${ }^{14}$ and commence a 48 hour multi-antibiotic regimen (dependent on recent sensitivity testing) similar to that used by the Toronto transplant group. ${ }^{15}$ We have successfully transplanted two ET-12 infected patients with current survival times of up to 1 year. The antibiotic regimens used were aztreonam, clindamycin, gentamicin, and systemic taurolidine $(250 \mathrm{ml} 2 \%$ taurolidine intravenously qds) in one patient and clindamycin, chloramphenicol, and temocillin with nebulised taurolidine in the other.

In conclusion, the ET-12 epidemic clone was the most prevalent strain in patients infected with $B$ cenocepacia referred to our unit for pre-transplant assessment. Although previously reported deaths following transplantation were associated with ET-12, successful transplantation of patients infected with this strain has been achieved using an altered management regimen. It remains to be determined whether these measures will provide safer management strategies for patients infected with other $B$ cenocepacia strains.

\section{Authors' affiliations}

A De Soyza, P A Corris, Transplantation and Immunobiology Group, The Freeman Hospital, High Heaton, Newcastle upon Tyne NE7 7DN, UK

K Morris, L Archer, J Perry, K Gould, Department of Medical Microbiology, The Freeman Hospital, High Heaton, Newcastle upon Tyne NE7 7DN, UK

C Doherty, J Govan, Department of Medical Microbiology, University of Edinburgh, Edinburgh EH8 9AG, UK

A McDowell, Biomolecular Sciences Group, School of Pharmacy, Queen's University, Belfast BT9 7BL, UK

Dr De Soyza received support via Breathe North and Wellcome Trust Clinical training fellowships.

\section{REFERENCES}

1 Isles A, Maclusky I, Corey M, et al. Pseudomonas cepacia infection in cystic fibrosis: an emerging problem. J Pediatr 1984;104:206-10.

2 Mahenthiralingam E, Baldwin A, Vandamme P. Burkholderia cepacia complex infection in patients with cystic fibrosis. J Med Microbiol 2002:51:533-8.

3 Vandamme P, Holmes B, Coenye T, et al. Burkholderia cenocepacia sp nova new twist to an old story. Res Microbiol 2003;154:91-6.

4 De Soyza A, McDowell A, Archer L, et al. Burkholderia cepacia complex genomovars and pulmonary transplantation outcomes in patients with cystic fibrosis. Lancet 2001;358:1780-1.

5 Aris RM, Routh JC, LiPuma JJ, et al. Lung transplantation for cystic fibrosis patients with Burkholderia cepacia complex. Survival linked to genomovar type. Am J Respir Crit Care Med 2001 ; 164:2102-6.

6 Sajian U, Wu Y, Kent G, et al. Preferential adherence of cable-piliated Burkholderia cepacia to respiratory epithelia of CF knockout mice and human cystic fibrosis lung explants. J Med Microbiol 2000;49:875-85.

7 Mahenthiralingam E, Simpson DA, Speert DP. Identification and characterization of a novel DNA marker associated with epidemic Burkholderia cepacia strains recovered from patients with cystic fibrosis. J Clin Microbiol 1997;35:808-16.

8 Agodi A, Barchitta M, Giannino V, et al. Burkholderia cepacia complex in cystic fibrosis and non-cystic fibrosis patients: identification of a cluster of epidemic lineages. J Hosp Infect 2002;50:188-95

9 Hasan A, Corris PA, Healy $M$, et al. Bilateral sequential lung transplantation for end stage septic lung disease. Thorax 1995;50:565-6.

10 Mahenthiralingam E, Bischof B, Byrne SK, et al. DNA-based diagnostic approaches for the identification of Burkholderia cepacia complex, Burkholderia vietnamiensis, Burkholderia multivorans, Burkholderia stabilis, and Burkholderia cepacia genomovars I and III. J Clin Microbiol 2000;38:3165-73.

11 Saijan US, Sun L, Goldstein R, et al. Cable (cbl) type II pili of cystic fibrosisassociated Burkholderia (Pseudomonas) cepacia: nucleotide sequence of the cblA major subunit pilin gene and novel morphology of the assembled appendage fibers. J Bacteriol 1995; 177:1030-8.

12 Steinbach S, Sun L, Jiang RZ, et al. Transmissibility of Pseudomonas cepacia infection in clinic patients and lung-transplant recipients with cystic fibrosis. N Engl J Med 1994;331:981-7.

13 Heath DG, Hohneker K, Carriker C, et al. Six-year molecular analysis of Burkholderia cepacia complex isolates among cystic fibrosis patients at a referral center for lung transplantation. J Clin Microbiol 2002;40:1 188-93.

14 Perry JD, Riley G, Johnston S, et al. Activity of disinfectants against gramnegative bacilli isolated from patients undergoing lung transplantation for cystic fibrosis. J Heart Lung Transplant 2002;21:1230-1.

15 Chaparro C, Maurer J, Gutierrez C, et al. Infection with Burkholderia cepacia in cystic fibrosis; outcome following lung transplantation. Am J Respir Crit Care Med 2001;163:43-8. 